\title{
Drug-Induced Sleep Endoscopy Changes the Treatment Concept in Patients with Obstructive Sleep Apnoea
}

\author{
Jaroslava Hybášková, ${ }^{1}$ Ondřej Jor, ${ }^{2}$ Vilém Novák, ${ }^{3}$ Karol Zeleník, ${ }^{1,4}$ \\ Petr Matoušek, ${ }^{1,4}$ and Pavel Komínek ${ }^{1,4}$ \\ ${ }^{1}$ Department of Otorhinolaryngology and Head and Neck Surgery, University Hospital Ostrava, Ostrava, Czech Republic \\ ${ }^{2}$ Department of Anesthesiology, Resuscitation and Intensive Medicine, University Hospital Ostrava, Ostrava, Czech Republic \\ ${ }^{3}$ Department of Pediatric Neurology, University Hospital Ostrava, Ostrava, Czech Republic \\ ${ }^{4}$ Faculty of Medicine, University of Ostrava, Ostrava, Czech Republic
}

Correspondence should be addressed to Jaroslava Hybášková; jaroslava.hybaskova@fno.cz

Received 3 August 2016; Revised 14 November 2016; Accepted 23 November 2016

Academic Editor: Francesco Doglietto

\begin{abstract}
Copyright ( 2016 Jaroslava Hybášková et al. This is an open access article distributed under the Creative Commons Attribution License, which permits unrestricted use, distribution, and reproduction in any medium, provided the original work is properly cited.
\end{abstract}

\begin{abstract}
The present study evaluated whether drug-induced sleep endoscopy (DISE) helps identify the site of obstruction in patients with obstructive sleep apnoea (OSA). A total of 51 consecutive patients with polysomnography-confirmed OSA were enrolled in this prospective study. The presumed site of obstruction was determined according to history, otorhinolaryngologic examination, and polysomnography and a therapeutic plan designed before DISE. In 11 patients with severe OSA and/or previously failed continuous positive airway pressure (CPAP) treatment, DISE with simultaneous CPAP was performed. Multilevel collapse was noted in 49 patients (96.1\%). The most frequent multilevel collapse was palatal, oropharyngeal, and tongue base collapse $(n=17,33.3 \%)$, followed by palatal and oropharyngeal collapse $(n=12,23.5 \%)$. Pathology of the larynx (epiglottis) was observed in 16 patients (31.4\%). The laryngeal obstruction as a reason for intolerance of CPAP was observed in 3/11 (27.3\%) patients. After DISE, the surgical plan was changed in 31 patients $(60.8 \%)$. The results indicate that DISE helps identify the site of obstruction in the upper airways in patients with OSA more accurately and that the larynx plays an important role in OSA.
\end{abstract}

\section{Introduction}

The main diagnostic challenge in patients with obstructive sleep apnoea (OSA) is to determine the location of the obstruction in the upper airway during sleep [1], as the otorhinolaryngologic examination used to determine the probable site is performed when the patient is awake. Therefore, drug-induced sleep endoscopy (DISE) was introduced into the diagnostic algorithm for OSA patients during the last decade $[2,3]$. The advantage of DISE is that collapse of the upper airways not visible when awake can be observed during sleep and therapy targeted according to the location and degree of obstruction of the upper airway $[4,5]$. This approach would result in greater treatment success in patients with OSA.

Continuous positive airways pressure (CPAP) ventilation is currently accepted worldwide as the gold standard treatment in patients with severe OSA [2-8]. However, many patients do not tolerate CPAP due to laryngeal collapse, which in many cases can be diagnosed only during DISE. Therefore, DISE with CPAP can be helpful in identifying the reason for CPAP intolerance in patients with OSA [9].

The aim of this study was to present how DISE changes the therapeutic strategy in patients with OSA and highlight the role of the larynx in OSA.

\section{Materials and Methods}

This prospective study was approved by the Institutional Ethics Committee and performed in accordance with the Declaration of Helsinki, good clinical practice, and applicable regulatory requirements. The study was registered at ClincialTrials.gov (identifier NCTNCT02855515). Informed 
TABLE 1: Indication and contraindication for drug-induced sleep endoscopy.

\begin{tabular}{ll}
\hline & (i) $18-65$ years old \\
& (ii) OSA (mild, moderate AHI $\leq 25)$ \\
Indication & (iii) Severe OSA $(\mathrm{AHI} \geq 25)$, failed CPAP \\
& treatment \\
& (vi) Severe OSA $(\mathrm{AHI} \geq 25)$, patients who \\
& refused CPAP \\
\hline & (i) ASA 4 \\
Contraindication & (ii) Pregnancy \\
& (iii) Propofol or midazolam allergies
\end{tabular}

OSA, obstructive sleep apnoea; AHI, apnoea-hypopnoea index; ASA, American Society of Anesthesiologists.

written consent was obtained from all participants before any procedure was performed. The inclusion criteria were age 1864 years, snoring, and suspected OSA treated at the university hospital between August 2015 and July 2016.

2.1. History, Otorhinolaryngology, and Polysomnography. Patient history was taken with an emphasis on snoring and OSA symptoms. Daytime sleepiness was assessed using the Epworth Sleepiness Scale. Patients were also asked to grade the severity of snoring on a 10-point visual analogue scale $(0$ $=$ no snoring, $10=$ extreme snoring). Body mass index (BMI) was calculated. Otorhinolaryngologic examination focused on nasal, pharyngeal, and laryngeal pathologies (e.g., uvula enlargement, soft palate webbing, enlargement of the palatal tonsils, hypertrophy of the base of the tongue, visibility of the vallecula, and pathology of the epiglottis). Each patient then underwent polysomnography and was divided into one of three groups according to OSA severity: mild $(5<$ apnoeahypopnoea index $(\mathrm{AHI}) \leq 15)$, moderate $(15<\mathrm{AHI} \leq 25)$, or severe (AHI $>25$ events per hour of sleep).

The presumed site of obstruction was determined based on the results of the examination and a therapeutic plan designed. Patients with severe OSA were sent for CPAP treatment, whereas surgery was recommended for patients with mild and moderate OSA.

2.2. Drug-Induced Sleep Endoscopy. The indications and contraindications for DISE are shown in Table 1. Patients were premedicated with a combination of $5 \mathrm{mg}$ midazolam and $0.5 \mathrm{mg}$ atropine intramuscularly 30 minutes before DISE. Patients were lying in a supine position on an operating table, the lights were dimmed, and the room was quiet to minimize stimuli. Local anesthesia was administered in the nasal cavity as two sprays of $10 \%$ lidocaine into each nostril. Patients were monitored by electrocardiogram (ECG), blood oxygen saturation, and noninvasive blood pressure, and the depth of anesthesia was monitored by bispectral index (BIS). Anesthesia was induced by an intravenous bolus of $1 \mathrm{mg}$ propofol per $\mathrm{kg}$ of ideal body weight and maintained by repeated boluses of $10-20 \mathrm{mg}$ propofol every $3-5$ minutes for a target BIS of 50-70. After the patient reached a satisfactory level of sedation, a flexible fiber optic intubation scope $(3.7 \mathrm{~mm} \times 65 \mathrm{~cm})$ coated with AntiFog spray Aplex was introduced into the nasal cavity to observe the nasal passage,

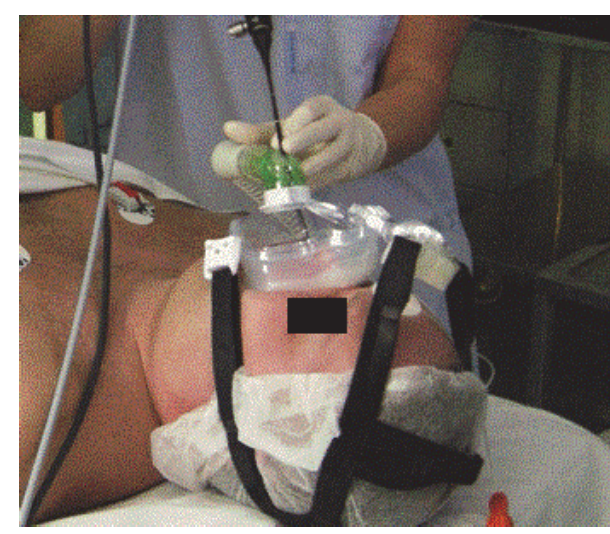

FIGURE 1: Drug-induced sleep endoscopy with CPAP.

nasopharynx, velum, oropharynx (including tonsils), tongue base, epiglottis, and larynx. The examination duration was at least 15 minutes in order to detect the obstruction. DISE findings were reported using the VOTE classification system according to Kezirian et al. [10, 11].

At the end of DISE in patient with tongue base obstruction a jaw thrust (or Esmarch maneuver) was performed. The mandible was gently advanced up to $4-5 \mathrm{~mm}$. The maneuver was evaluated as successful when enlargement of the upper airway was observed and apnoea disappeared as with mandibular repositioning appliance (MRA) or tongue advancing surgery [12].

In 11 patients with severe OSA and/or previously failed CPAP treatment, DISE with simultaneous CPAP was performed. The CPAP mask was placed on the face and flexible endoscopy performed through the mask (Figure 1). The velum, oropharynx (including tonsils), tongue base, epiglottis, and larynx were observed under $6-20 \mathrm{~cm} \mathrm{H}_{2} \mathrm{O}$ pressure and changes in the lumen compared with/without the overpressure, allowing the collapsibility of soft tissues to be evaluated.

After finishing DISE, patients were awakened to full consciousness in the operating room and then transferred to a recovery room for observation for 1 hour.

2.3. Evaluation of Changes in Indication for the Site of Surgery. The indications for surgery according to otorhinolaryngology before DISE and after DISE were compared. For statistical analysis, descriptive statistic (arithmetic mean, standard deviation, and frequency tables) was used.

\section{Results}

A total of 185 patients were evaluated for snoring and suspected OSA at our department during the study period. Only 51 patients ( 4 women, 47 men) were indicated for DISE and included in the statistical analysis (Table 2). The mild form of OSA was observed in 14 patients (27.4\%), moderate OSA in 11 patients $(21.6 \%)$, and severe OSA in 26 patients (51.0\%).

The most common observation was partial or complete palatal collapse, followed by oropharyngeal collapse, tongue 
TABLE 2: Baseline patient characteristics.

\begin{tabular}{lc}
\hline Variable & All subjects $(N=51)$ \\
\hline Males & $47(92.2 \%)$ \\
Age, years & $44.7 \pm 11.3$ \\
BMI, kg/m ${ }^{2}$ & $30.1 \pm 4.0$ \\
AHI, per hour sleep & $28.4 \pm 16.6$ \\
\hline
\end{tabular}

Data are presented as $n(\%)$ or mean \pm SD. BMI, body mass index; AHI, apnoea-hypopnoea index.

TABLE 3: Type of obstruction in the upper airway.

\begin{tabular}{lc}
\hline Type of collapse in upper airway & Number of patients $(N=51)$ \\
\hline Palatal collapse & $50(98.0 \%)$ \\
(i) Concentric & $27(54.0 \%)$ \\
(ii) Anteroposterior & $21(42.0 \%)$ \\
(iii) Laterolateral & $2(4.0 \%)$ \\
Oropharyngeal collapse & $45(88.2 \%)$ \\
(i) Concentric & $1(2.2 \%)$ \\
(ii) Laterolateral & $44(97.8 \%)$ \\
Tongue base collapse & $31(60.8 \%)$ \\
(i) Anteroposterior & $31(100.0 \%)$ \\
Epiglottis collapse & $16(31.4 \%)$ \\
(i) Laterolateral & $2(12.5 \%)$ \\
(ii) Anteroposterior & $14(87.5 \%)$ \\
\hline
\end{tabular}

TABLE 4: Type of multilevel collapse in the upper airway.

\begin{tabular}{lc}
\hline Type of multilevel collapse & Number of patients \\
\hline Palatal + base of tongue & $4(7.9 \%)$ \\
Palatal + base of tongue + epiglottis & $1(2.0 \%)$ \\
Palatal + oropharyngeal & $12(23.5 \%)$ \\
Palatal + oropharyngeal + epiglottis & $6(11.8 \%)$ \\
Palatal + oropharyngeal + base of tongue & $17(33.3 \%)$ \\
$\begin{array}{l}\text { Palatal + oropharyngeal + base of tongue + } \\
\text { epiglottis }\end{array}$ & $9(17.6 \%)$
\end{tabular}

base collapse, and epiglottis collapse (Table 3). The most frequent type of palatal collapse was concentric collapse and then anteroposterior collapse. Regarding the larynx, two types of epiglottis collapse were observed: anterior-posterior and laterolateral (Table 3).

Multilevel collapse occurred in 49 of the 51 patients (96.1\%) (Table 4). Collapse in two locations was observed in 16 patients (31.4\%), in three locations in 24 patients (47.1\%), and in all levels (palatal, oropharyngeal, tongue base, and epiglottis) in 9 patients (17.6\%). The most frequent multilevel collapse pattern was a combination of palatal, oropharyngeal, and base of tongue collapse, followed by a combination of palatal and oropharyngeal collapse (Table 4).

In 11 patients with severe OSA and/or previously failed CPAP treatment, DISE with simultaneous CPAP was performed. Multilevel collapse (two and more locations) occurred in 11 patients (100\%), in three locations in 5/11 (45.5\%) patients, and in all levels in 3/11 (27.3\%) patients. The
TABLE 5: Change in indication to operation after drug-induced sleep endoscopy (DISE).

\begin{tabular}{|c|c|c|}
\hline $\begin{array}{l}\text { Indication for } \\
\text { surgery in } \\
\text { wakefulness } \\
\text { after ENT } \\
\text { examination }\end{array}$ & $\begin{array}{l}\text { Indication for surgery after } \\
\text { DISE (jaw thrust was done } \\
\text { as a part of DISE in all } \\
\text { patients) }\end{array}$ & $\begin{array}{l}\text { Number } \\
\text { of patients } \\
(N=31)\end{array}$ \\
\hline UPPP & $\mathrm{UPPP}+\mathrm{RFBT}$ & $7(22.6 \%)$ \\
\hline UPPP & Epiglottopexy & $1(3.2 \%)$ \\
\hline UPPP & UPPP + epiglottopexy & $2(6.4 \%)$ \\
\hline UPPP & $\begin{array}{l}\text { UPPP + RFBT + } \\
\text { epiglottopexy }\end{array}$ & $1(3.2 \%)$ \\
\hline RFBT & UPPP & $1(3.2 \%)$ \\
\hline RFBT & Mandibular advancement & $1(3.2 \%)$ \\
\hline $\begin{array}{l}\mathrm{UPPP}+ \\
\text { RFBT }\end{array}$ & UPPP & $8(25.1 \%)$ \\
\hline $\begin{array}{l}\mathrm{UPPP}+ \\
\mathrm{RFBT}\end{array}$ & UPPP + epiglottopexy & $3(9.7 \%)$ \\
\hline $\begin{array}{l}\mathrm{UPPP}+ \\
\mathrm{RFBT}\end{array}$ & $\begin{array}{l}\mathrm{UPPP}+\mathrm{RFBT}+ \\
\text { epiglottopexy }\end{array}$ & $4(12.9 \%)$ \\
\hline $\begin{array}{l}\text { UPPP + } \\
\text { RFBT }\end{array}$ & RFBT + epiglottopexy & $1(3.2 \%)$ \\
\hline $\begin{array}{l}\text { UPPP + } \\
\text { RFBT }\end{array}$ & Mandibular advancement & $1(3.2 \%)$ \\
\hline
\end{tabular}

UPPP, uvulopalatopharyngoplasty; RFBT, radiofrequency base of the tongue.

laryngeal obstruction as a reason for intolerance of CPAP was observed in 3/11 (27.3\%) patients.

After DISE, the treatment concept was changed in 31 patients $(60.8 \%)$. The most common recommendation was to change uvulopalatopharyngoplasty (UPPP) with radiofrequency of tongue base (RFTB) to UPPP alone. The second most frequent change was from UPPP to UPPP + RFTB. Due to pathology of the larynx, 4 of 31 patients (12.9\%) with an indication for UPPP + RFTB had the procedure changed to UPPP + RFITT + epiglottopexy (Table 5).

\section{Discussion}

OSA is the most common type of sleep-disordered breathing, with a prevalence of $2-4 \%$ in the adult population and an increasing rate of morbidity and mortality $[5,8]$. During wakefulness, collapse of the upper airways is prevented by high pharyngeal neuromuscular tone. Reduction of this neurophysiological phenomenon with consequent hypotonia of the upper airways is an important factor in the pathophysiology of OSA [2].

The main diagnostic challenge in patients with OSA is to determine the location of the obstruction of the upper airway during sleep [1]. DISE allows examination of the dynamic status of upper airways during sleep and determining the site and pattern of obstruction (anteroposterior, lateral, or concentric) [3]. Obstruction of the upper airways in OSA is usually located at multiple levels, frequently requiring multilevel surgery. With precise identification, surgery can be targeted and greater treatment success achieved [5]. Another 


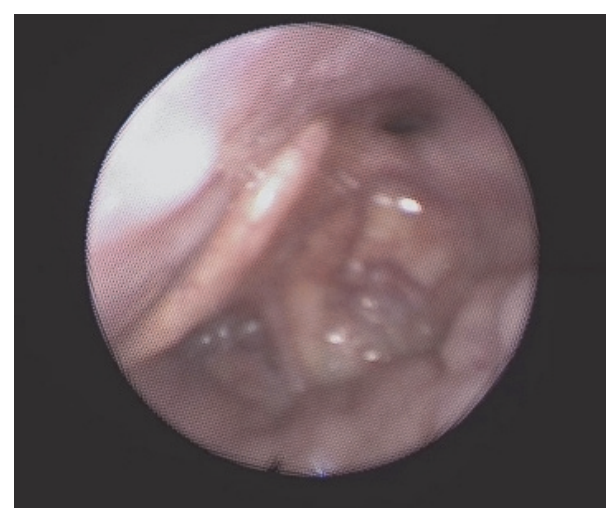

FIGURE 2: Collapsed epiglottis on the posterior wall of the hypopharynx during drug-induced sleep endoscopy. The condition caused apnoea.

advantage of DISE is that a jaw thrust (or Esmarch maneuver) can be performed. The modified jaw thrust maneuver is fast and simple and can simulate mandibular advancement as expected with MRA or tongue advancing surgery $[6,12]$. Tongue base collapse (anterior-posterior) in our study group was observed in $31(60.8 \%)$ patients. Change of treatment after DISE with simultaneous jaw thrust maneuver as a part of DISE is summarized in Table 5.

Vroegop et al. reported on 1249 patients who underwent DISE [13]. In their study, palatal collapse was observed in $81.0 \%$ of patients. Multilevel collapse was observed in $68.2 \%$ of patients; the most frequent combination was palatal and tongue base collapse (25.5\% of patients). The prevalence of complete collapse, multilevel collapse, and hypopharyngeal collapse increased with increasing severity of OSA [14]. In a smaller study, Pilaete et al. performed DISE in 61 patients and observed multilevel collapse in 48 (78.8\%) patients. They reported that, after DISE, the initial management plan changed in $41 \%$ of all patients [15]. In addition, Salamanca et al. reported that oropharyngeal obstruction was present in $96.0 \%$ of 641 patients who underwent DISE and multiple obstructions were present in $50.0 \%$ [16]. In the present study, the most common observation was partial or complete palatal collapse (98.0\% of patients) and multilevel collapse was observed in $96.1 \%$ of patients.

As pathology of the larynx (epiglottis) was observed in $31.4 \%$ of patients, the role of the larynx in OSA can be concluded to be significant and often underdiagnosed. The reason is that collapse of the epiglottis is not usually seen when the patient is awake. Furthermore, laryngeal obstruction may be a reason for intolerance of CPAP and failure of OSA surgery [9]. The most common type of epiglottis pathology in our study group was closing door epiglottis, when the epiglottis collapsed on the posterior wall of the hypopharynx during inspiration (Figure 2). When awake, laryngeal obstruction may be hypothesized to be due to the deformed epiglottic shape, but direct visualization of laryngeal collapse is possible only during sedation.

Civelek et al. demonstrated that the majority of adult OSA patients unable to tolerate CPAP have multilevel obstruction [9], and 15\% had primary epiglottic obstruction in the hypopharynx. Primary epiglottic obstruction may not be amenable to CPAP, as positive pressure may further push a retroflexed epiglottis into the endolarynx [14].

In our study, DISE with CPAP was performed in 11 patients. Just the pathology of epiglottis (closing door) was the reason for CPAP failure in 3/11 (27.3\%). The pressure for opening UA (upper airway) was in two patients $12 \mathrm{hPa}$ and in one patient $18 \mathrm{hPa}$. When the pressure was lower, the epiglottis was pushed into the endolarynx.

Thus, to better understand the pathophysiology of airway collapse, examination by DISE with CPAP can be beneficial and more studies are needed.

After DISE, the surgical plan changed in $60.8 \%$ of all patients in our study. This is similar to the results of others studies. The most frequent change in indication was from UPPP + RFTB to UPPP alone. In these cases we observed that hypertrophy of the tongue base during wakefulness does not mean that it is the cause of airway obstruction during sleep.

A high percentage of larynx obstruction may be the reason for intolerance of CPAP and failure of OSA surgery. Before DISE there were limited options for examining laryngeal pathology and, therefore, surgery did not focus on this site.

\section{Conclusion}

DISE has an emerging role in identifying the site and characteristics of obstructions associated with OSA. The procedure provides important information about dynamic changes in the upper airways during sleep. Moreover, DISE with simultaneous CPAP may be used for the titration of CPAP. DISE has already revealed that the larynx plays a more important role in OSA than previously assumed. Laryngeal obstruction may be a cause of intolerance of CPAP and failure of OSA surgery. To better understand the pathophysiology of airway collapse, more prospective studies of DISE with CPAP may be beneficial.

\section{Competing Interests}

The authors declare that there is no actual or potential conflict of interests in relation to this article. No benefits in any form have been received or will be received from a commercial party related directly or indirectly to the subject of this article.

\section{Acknowledgments}

This paper was supported by the Ministry of Health, Czech Republic, Conceptual Development of Research Organization, MH CZ-DRO-FNOs/2016.

\section{References}

[1] O. Aktas, O. Erdur, A. A. Cirik, and F. T. Kayhan, "The role of drug-induced sleep endoscopy in surgical planning for obstructive sleep apnea syndrome," European Archives of Oto-RhinoLaryngology, vol. 272, no. 8, pp. 2039-2043, 2014. 
[2] A. Campanini, P. Canzi, A. De Vito, I. Dallan, F. Montevecchi, and C. Vicini, "Awake versus sleep endoscopy: personal experience in 250 OSAHS patients," Acta Otorhinolaryngologica Italica, vol. 30, no. 2, pp. 73-77, 2010.

[3] M. Cavaliere, F. Russo, and M. Iemma, "Awake versus druginduced sleep endoscopy: evaluation of airway obstruction in obstructive sleep apnea/hypopnoea syndrome," Laryngoscope, vol. 123, no. 9, pp. 2315-2318, 2013.

[4] E. De Corso, A. Fiorita, G. Rizzotto et al., "The role of druginduced sleep endoscopy in the diagnosis and management of obstructive sleep apnoea syndrome: our personal experience," Acta Otorhinolaryngologica Italica, vol. 33, no. 6, pp. 405-413, 2013.

[5] A. De Vito, M. Carrasco Llatas, A. Vanni et al., "European position paper on drug-induced sedation endoscopy (DISE)," Sleep and Breathing, vol. 18, no. 3, pp. 453-465, 2014.

[6] C. Eichler, J. U. Sommer, B. A. Stuck, K. Hörmann, and J. T. Maurer, "Does drug-induced sleep endoscopy change the treatment concept of patients with snoring and obstructive sleep apnea?" Sleep and Breathing, vol. 17, no. 1, pp. 63-68, 2013.

[7] E. Fernández-Julián, M. Á. García-Pérez, J. García-Callejo, F. Ferrer, F. Martí, and J. Marco, "Surgical planning after sleep versus awake techniques in patients with obstructive sleep apnea," Laryngoscope, vol. 124, no. 8, pp. 1970-1974, 2014.

[8] M. Friedman, Sleep Apnea and Snoring, Surgical and NonSurgical Therapy, Saunders/Elsevier, Beijing, China, 2009.

[9] S. Civelek, I. E. Emre, D. Dizdar et al., "Comparison of conventional continuous positive airway pressure to continuous positive airway pressure titration performed with sleep endoscopy," The Laryngoscope, vol. 122, no. 3, pp. 691-695, 2012.

[10] E. J. Kezirian, W. Hohenhorst, and N. De Vries, "Drug-induced sleep endoscopy: the VOTE classification," European Archives of Oto-Rhino-Laryngology, vol. 268, no. 8, pp. 1233-1236, 2011.

[11] E. J. Kezirian, D. P. White, A. Malhotra, W. Ma, C. E. McCulloch, and A. N. Goldberg, "Interrater reliability of drug-induced sleep endoscopy," Archives of Otolaryngology-Head and Neck Surgery, vol. 136, no. 4, pp. 393-397, 2010.

[12] E. De Corso, G. Bastanza, G. D. Marca et al., "Drug-induced sleep endoscopy as a selection tool for mandibular advancement therapy by oral device in patients with mild to moderate obstructive sleep apnoea," Acta Otorhinolaryngologica Italica, vol. 35, no. 6, pp. 426-432, 2015.

[13] A. V. Vroegop, O. M. Vanderveken, A. N. Boudewyns et al., "Drug-induced sleep endoscopy in sleep-disordered breathing: report on 1,249 cases," The Laryngoscope, vol. 124, no. 3, pp. 797802, 2014.

[14] M. J. L. Ravesloot and N. De Vries, "One hundred consecutive patients undergoing drug-induced sleep endoscopy: results and evaluation," Laryngoscope, vol. 121, no. 12, pp. 2710-2716, 2011.

[15] K. Pilaete, J. De Medts, and K. G. Delsupehe, "Drug-induced sleep endoscopy changes snoring management plan very significantly compared to standard clinical evaluation," European Archives of Oto-Rhino-Laryngology, vol. 271, no. 5, pp. 1311-1319, 2014.

[16] F. Salamanca, F. Constantini, A. Bianchi et al., "Identification of obstructive sites and pattwens in obstructive sleep apnoea synrome by sleep endoscopy in 614 patients," Acta Otorhinolaryngologica Italica, vol. 33, pp. 261-266, 2013. 


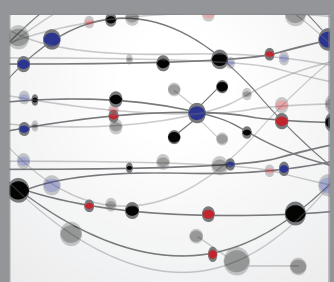

The Scientific World Journal
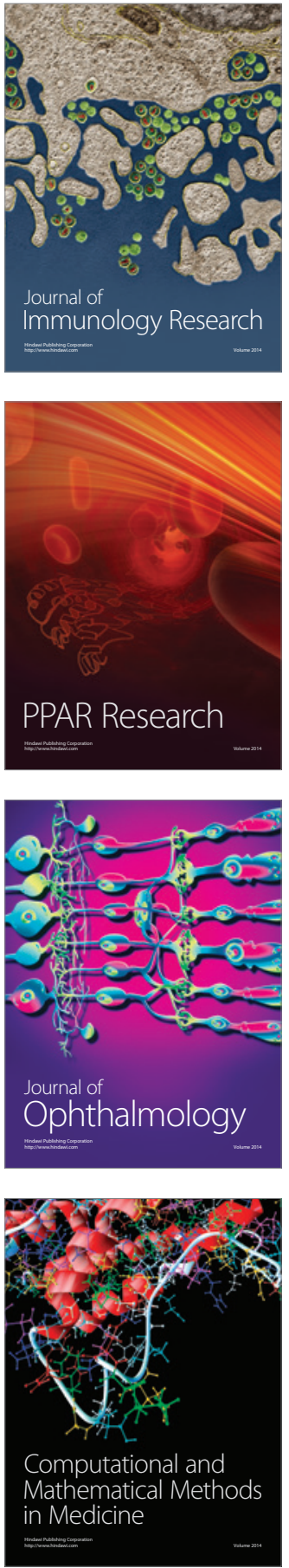

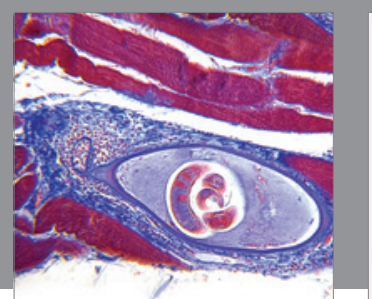

Gastroenterology Research and Practice

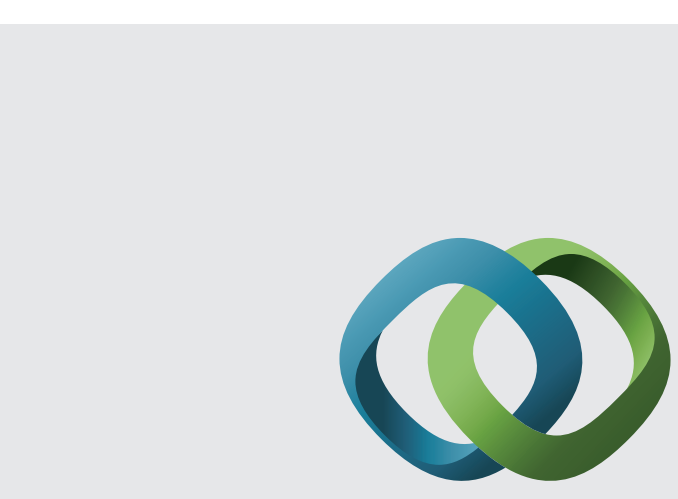

\section{Hindawi}

Submit your manuscripts at

http://www.hindawi.com
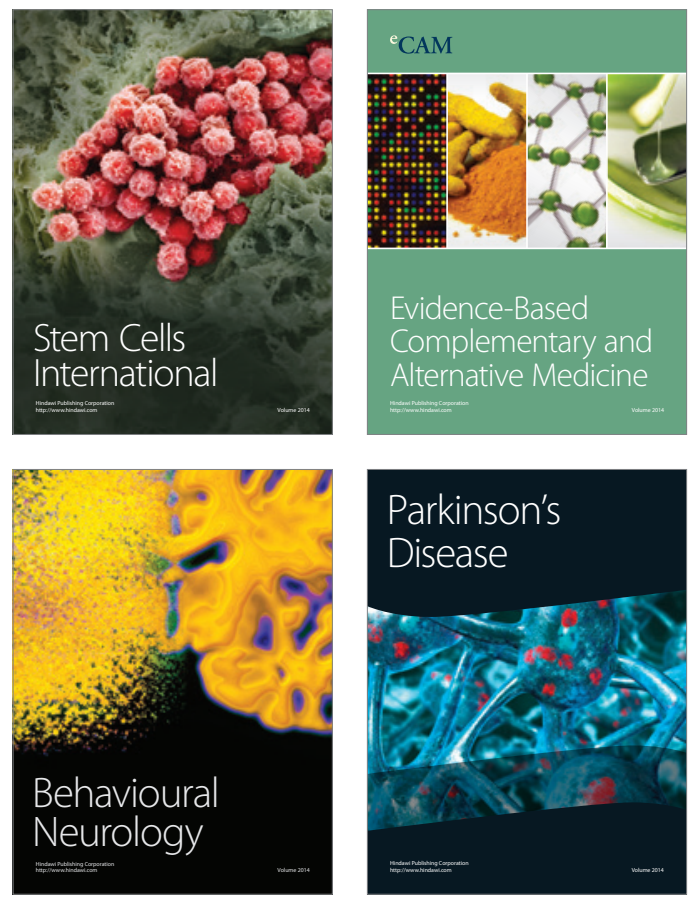
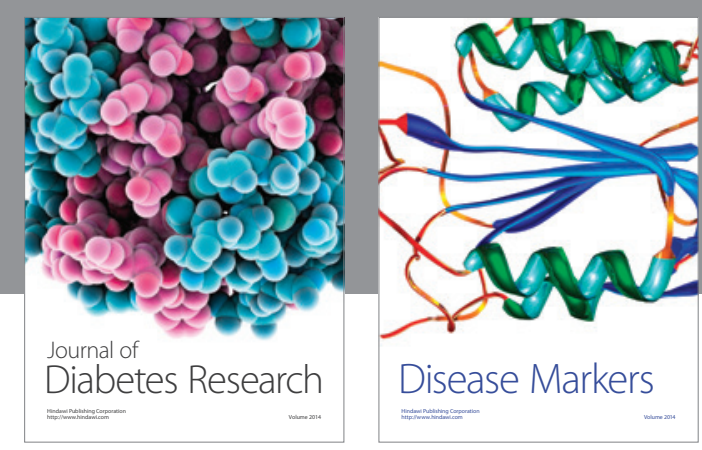

Disease Markers
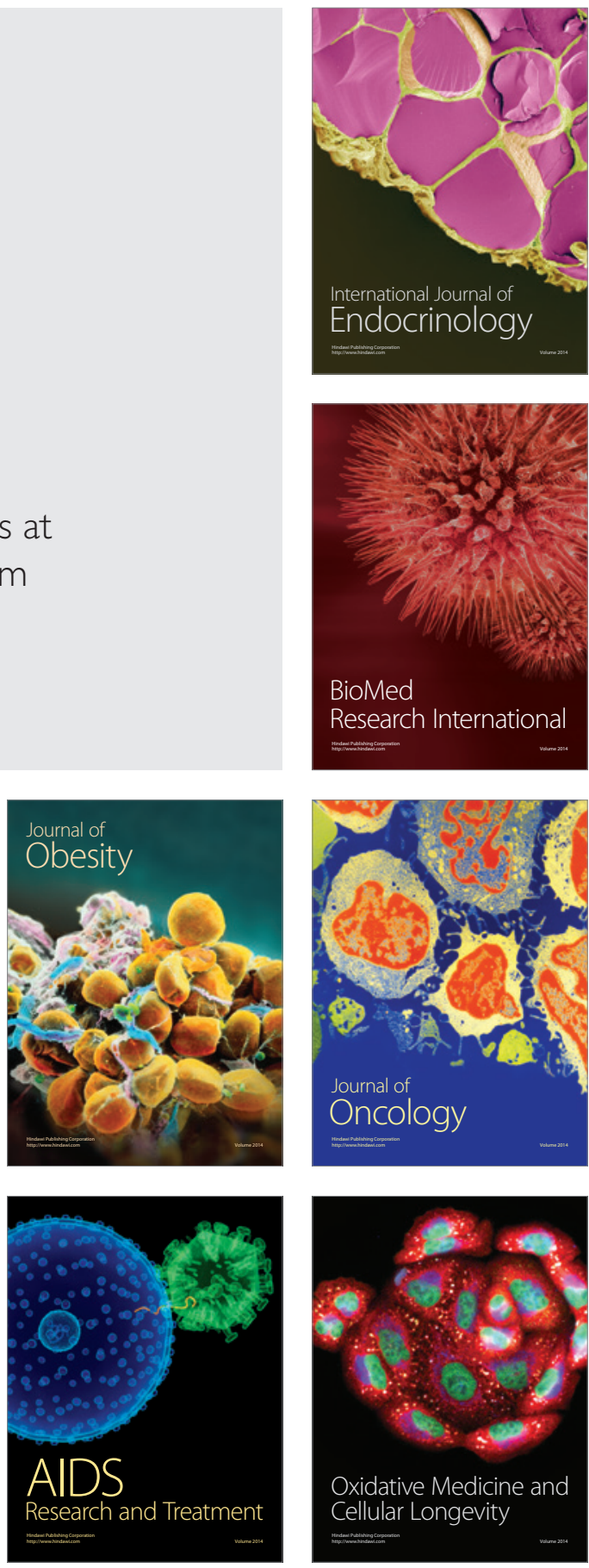